were removed. The tumours extended down to, and, as far as one could judge, were continuous with the periosteum over the tuberosities of the ischia along their whole length. The ensuing large cavities were flushed out with hot saline and obliterated with buried catgut sutures, the wounds being closed with interrupted silkworm gut. The smaller tumour over th sacrum was enucleated without any difficulty. 'The patient made an uninterrupted recovery, and was discharged to a convalescent home. He states that he lately sat down at a concert on a hard form for one and a half hours-a thing he has been unable to do for thirty years.

To the naked eye the masses appeared fibrous and avascular throughout. There was no obvious lamination or cavity in the tumours, which appeared to derive their blood supply from their capsules. It appeared remarkable that masses of such size and such a poor blood supply could survive without undergoing some obvious degenerative changes. Captain Campbell kindly examined these specimens for me, and reported that the tumours were typical fibromata. There has thus to be considered a case of widespread fibrous chainges occurring in bursae in normal positions and a fibroma occupying a situation not as a rule where one finds a normal bursa-I refer to the tumour of the left thumb.

The commonest changes in bursae set up by chronic irritation causing inflammation are seen in the bursitis of the occupation diseases known as housemaid's knee, miner's elbow, and weaver's bottom. These conditions are respectively an inflammation of the bursae in the prepatellar, olecranon, and ischial tuberosity situations. The condition starts as a hydrops. The bursae, irritated by prolonged pressure, secrete an excess of synovial fluid, Nature's method of forming a more efficient water cushion. Secondary infection, haemorrhage, deposition of fibrin substance, and thickening of the wall of the bursa may ensue.

The tumours in this case are unlike this condition. The youngest tumour is the small one in the thumb and the one removed from the sacrum; neither of these appears to have started as a hydrops or a haemorrhage. It is therefore unlikely that the larger ones started as either of these two conditions, afterwards undergoing solidification. The patient's occupation is not one that, so far as can be scen, is likely to give rise to bursitis. He has been at sea working in engine-rooms for thirty years, and apart from some cramping, does not do very sedentary work or work that throws prolonged strain on the situations involved. It appears, therefore, that the theory that the patient's occupation caused this condition can be dismissed.

The question now arises if this condition may be due to tubercle, syphilis, or gonorrhoea. Tubercle can be immediately dismissed. It is impossible to imagine chronic masses of this size without the presence of caseation or giant cell formation. In discussing the probable venereal origin of this condition we must go back to 1887 . In August of that year the patient developed a " running" which ceased after three weeks. He denies chancre, sore throats, rash, or other syphilitic manifestations. Almost immediately after cessation of this urethritis he was admitted to hospital in Bombay with what he deseribes as rheumatic fever, and states that he was in hospital till January, 1888. He has suffered from rheumatic pains since, but has never been in hospital again with them Twelve months after the illness he noticed that lumps were beginning to grow over the buttocks and elbows. At first as large as peas, they gradually and insidiously grew, till at the end of thirty years they have reached their present size. His only previous illness was typhoid fever in 1803, in Constantinople. The tumours five caused no pain or trouble, apart from those of the buttocks, which have prevented the patient from sitting down. Captain Mackey reports that the patient has a strongly positive Wassermann reaction. Must we look upon the fibroid changes in these bursae as being of a syphilitic or gonorrhoeal origin?

In thirty years the only syphilitic changes are these large deposits of fibrous tissue, without, as far as can be found, there having been any other syphilitic or gummatous manifestations taking place. Gonorrhoea certainly produces inflammation of synovial membrane, and therefore could produce a bursitis. I think it unlikely that it could produce the condition we are considering.

Taking into consideration the histology of these masses - the fact that one of them has probably occurred in tissue other than synovial membrane-it seems probable, as Erichsen points out, thet we have to consider a case in which the tissues have developed a tendency to undergo fibrous hyperplasia, the changes taking place chiefly in the scnovial walls of bursae. Admitting the syphilitic taint and the gonorrhoeal history, the rheumatic pains similar to the pain felt in nodes, I look upon these masses as primary non-malignant growths of fibrous tissue.

\section{BRONCHOSCOPY IN THE TREATMENT OF} ASTHMA.

BY W. S. SYME, M.D., F.R.S.E.,

BURGEON, EAR, NOBE, AND THROAT HOSPITAL, GLABGOW : EXTRAACADEMICAL LECTURER ON DISEAGES OF THE THROAT
AND NOSE, GLASGOW.

Among the patients who come under the care of those engaged in the special branch of rhinology and laryngology there are always a certain number who complain of asthmatic attacks of a more or less definite character. In most of these patients the asthma is not severe, and is rather of the nature of difficulty of breathing caused by nasal obstruction, or at any rate of an interference by this obstruction with the co-ordinated movements of which regular respiration is composed. There are others in whicl asthma, and sometimes severe asthma, is associated with a nasal affection of which obstruction is not a prominent feature. In accessory sinus disease, and especially in maxillary antral disease, asthma is not infrequently com. plained of. The cure of the sinus disease is, as a rule, followed by disappearance of, or at any rate by marked alleviation of, the asthmatic attacks. The relation of the sinus disease to the asthma is probably complex, and depends on the intermittent obstruction which the discharge from the sinus causes, the irritation of the discharge on the upper air passages, and possibly the action of the products of suppuration on the higher nerve centres.

In speaking of asthma one of course recognizes that it is not a disease but a symptoni, and that for its proper study an inquiry into practically all the organs of the body would be necessary. Even in what is known as pure spasmodic asthma the problem is not simple. The respiratory apparatus is not alone at fault. There is also a susceptible condition of the central nervous system which in the present state of our knowledge canuot be more definitely described. It is evident, therefore, that in the proper treatment of asthma there is room for the co. operation of the specialist and the physician. Treatment applied to the air passages alone has, then, limitations and is empirical.

Not every case of asthma associated with a nasal or sinus affection is cured or even appreciably benefited by the effective treatment of the nasal or sinus condition. Moreover, in many cases there is no nasal abnormality, if one may say so, though it has been cynically said, "It's a poor nose a nasal surgeon can't find something wrong with." The nasal treatment of asthma, or in other words the treatment by cauterization of the nasal septum, has in my experience given only temporary benefit.

During the past two years I have treated eight cases of asthma by the direct application of silver nitrate solution to the lining of the bronchi with the aid of the broncho. soope. 'The ages of the patients ranged from 10 to 53 . Except in the youngest, iu whom chloroform was used, the treatment was conducted under cocaine anaesthesia with a preliminary hypodermic injection of omnopon or morphine and atropine. The position of the patient was that which-I prefer for bronchoscopy-lying on the left side with the head thrown back. The bronchoscope is introduced, and a mixture of cocaine and adrenalin is applied to the mucous membrane in advance of the tube as far as the openings of the secondary bronchi. The entrance to the secondary bronchi and the main bronchi are then swabbed with a 10 per cent. solution of silver nitrate. I have been specially careful to apply the solution to the regions of the bifurcations, because it seemed to me that the mucous membiane at these places was hyperaemic, and, at any rate, one would expect the air current to impinge most strongly at these points, and the reflex to be most active there. The idea underlying the treatment is that in asthma one factor in the causation is an exaggeration of a normal reflex. When an irritative substance, such as an irritating gas, touches the lining of the bronchi and larger branches, the smaller bronchi and bronclioles are by reflex action contracted for protective purposes. In attempting to weaken this reflex in asthma
by applications to the peripheral pole, it is not implied 
that the hypersensitiveness is at that part. Probably it is, as has been previously observed, central in the main. Silver nitrate solution suggested itself because it is com. monly used in irritative conditions of the higher parts of the respiratory apparatus, and because it leaves a coating of silver chloride or albuminate, and so its action is prolonged.

A fairly acute reaction follows the application, as would be expected, and the breathing is somewhat laboured for a day or two, during which the patient is kept in bed.

From the short notes of the cases treated, which are appended, it will be seen that the method is worthy of an extended trial. Indeed in several of the cases, and those severe, the results have been surprisingly good-quite, I am bound to say, beyond my expeciation.

\section{CASE I.}

Male, aged 44. Severe spasmodic asthma of many years duration. Has had various forns of treatment, including residence abroad, but without benefit. Lately the attacks have been more frequent and very severe, occurring every night and of 1 requiring morphine. He came under my care two years ago. He was then treated by the method described and the treatment was repeated on four occasions, the last being over ten months ago. At that time he reported that he had not had
such a good year for years. Now he reports that he has been practically free from asthma for the past year. He is busily engaged in Government work, and once he had to pass through a workshop where there were gas fumes, and, as a result, he had in the period. His general appearance reflects the improvement in his asthma.

\section{Case II.}

Male, aged 53. Frequent asthmatic attacks, especially in frosty weather. He was treated on one occasion eighteen months ago. The interesting point in his case was the discovery of a web formation in the shape of a firm band at the entrance to the right bronchus. He reports that his asthma has not benefited.

\section{CASE III.}

Male, aged 10. Bronchial asthma. This boy had gevere bronchisl astbma for-geveral years. There was no nasat or nasopharyngeal abnormal condition. Treated under chloroform more than a year ago. The mucous membrane of the bronchi, especially of the left, was oedematous. There was trouble with the anaesthetic and the applications of the silver nitrate were not so systematically carried out. For some time afterwards the breathing was better and he had no definite asthmatic attack, but now the asthma has returned. His father was advised to bring him back to have the treatment repeated, but has failed to do so.

\section{CASE IV.}

Male, aged 35. This man, a railway employee, had suffered from severe spasmodic asthma for years. He had been treated in various ways, by nasal cauterization among others, but in various ways, by nasal cauterization among others, but without benefit. Latterly he had become so much worse that
he was rendered unfit for work for about a week every month he was rendered unfit for work for about a week every month and was in danger of losing his post. More than a year ago he was treated by this method, the applications being made on the exception of one attack, which lept him away from work for a day, he has been quite free from asthma. He certainly looks much improved in health.

\section{Case v.}

Female, aged 24. First seen August 18th, 1916. During the previous four months she had had an attack of severe siasmorlic asthma every fortnight. Treated September 15th. She reports that she has been quite free from astlima since.

\section{Case vi.}

Female, aged 53. Bronchial asthma of some years' duration. Successfully operated on for right maxillary antral disease, but without benefit to the asthma. Treated September 15th. This was followed by a somewhat prolonged, though not severe, bronchial attack. She reports that she has been free from asthma for months.

\section{Case vir.}

Female, aged 24. Spasmodic asthma and paroxysmal sneezing for two or three years. Four severe asthmatic attacks recently lasting for some hours. Treated September 19th. A smal warty growth was seen on the wall of the left bronchus low down. She reports that she has had several asthmatic attacks during the winter, but for the past two months has been free.

\section{CASE VIII.}

Female, aged 18. Has suffered from severe spasmodic asthma since four years of age. Latterly the attacks recurred about every second week and left her unfit for work for a day or two. Her tonsils, which were enlarged, were enucleated without benefit tonsils, which were enlarged, were enucleated without benefit to the asthma. Treated by the application of silver nitrate with the aid of the bronchoscope on January 19th, 1917. She reports in health.

\section{A NEW CULTURE MEDIUM FOR THE GONOCOCCUS. \\ (Preliminary Note.)}

By Captain D. ThOMSOÑ, R.A.M.C., M.B., Ch.B.Edin., D.P.H.CANTAB.

THE following work was carried out in the laboratory of the Military Hospital, Rochester Row, London, S.W. under Lieut.-Colonel L. W. Harrison, D.S.O., R.A.M.C., and I wish to express here my keen appreciation of his encouragement and help.

There has been a great need recently for a satisfactory culture medium for the gonococcus, not only for diaguostic purpeses, but also for the manufacture of gonococcal vaccines. The media chiefly employed so far', namely, trypsinized pea extract and blood agar, have the following detects :

1. The growth is by no means profuse.

2. The medium is not clear and transparent, the trypsinized pea extract leing yellowish and opaque, aud the blood agar opaque and dark red. The whitish and translacent gonococcus growth is not seen clearly on such medis.

3. For vaccine purposes blood agar is very unsatisfactory because the water of condensation at the bottom of the tabe contains a dark brown débris of broken-down red cells. The gonococcus emulsion mixed with this water of condensation is in consequeuce coloured and full of débris, which renders it difficult to standardize.

4. The emulsion obtained from the trypsinized pea extract cultures, though yellow in colour, is quite satisfactory from this point of view. This culture medium, however. does not appeal to me as a natural one, and it is possible that after appeal to me as a natural one, and it is possible that after frequent subculture the gonococcus may lose its antigenic any rate, it seems reasonable to suppose that the best medium any rate, it seems reasonable to suppose that the
would be one which was as natural as possible.

My first attempt was to remove the great disadvantage of the blood agar (a natural medium) by using human blood minus the red corpuscles-that is, plasma. It was found that the gonococci grew as well on plasma agar in the proportion of 1 of plasma to 4 of agar as they did when the corpuscles were present.

The growth, however, was, as in the case of the blood agar medium, not profuse, but after some further experiments very strong growths were obtained with a similar nedium made up as follows:

1. Prepare nutrient agar ( 2.5 per cent.) in the ordinary way with bouillon and Witte's peptone (1 per cent.), and render it +6 acid.

2. Instead of adding to this 0.5 per cent. sodium chloride as is usual, add all the salts nataral to the humau blood (as in Ringer's solution)-namely : Sodium chloride 9 grams, calcium chloride 0.25 gram, and potassium chloride 0.42 gram per litre.

3 . Add glucose 2.5 per cent. This addition in some manner renders the growth much more profuse.

4. The uutrient agar with salts and glucose is then tubed, about $4 \mathrm{c.cm}$. being added to each test tube.

5. The sterile tubed agar is melted in boiling water, and after allowing it to cool to about $50^{\circ} \mathrm{C}$., add 1 c.cm. of human plasms to each tube and mix thoroughly by rolling the tube between the palms. Allow the medium to solidify in a sloping position.

For platiug, the contents of three tubes may be added to a Petri dish.

Method of Obtaining Human Plasma.

In all venereal hospitals blood is frequently drawn off fiom the veins of syphilitic * patients into test tubes for the Wassermann test. In consequence, the source of human plasma is always available.

When a supply of plasma is required, draw off three-quarter of a test-tube-full of blood with all sterile precautions. Have a sterile centrifugal tube ready, containing $2 \mathrm{c.cm}$, of a 2 per cent. solution on sodium citrate. Fill up this centrifugal tube with the freshly drawn blood. Plug it with a sterile cork (keep the corks in atcohol and barn off the alcohol before plugging) and centrifugalize. When the corpuscles are driven down, pipette off the supernatant plasma with a sterile $10 \mathrm{ccm}$. pipette and add l c.cm. to each tube of agar as inclicated above. If the test tube of blood is three-guarters full there is sufficient left for the Wassermann test after filling the centrifugal tube.t

The medium is therefore as easy to prepare as the blood agar itself, and it is certainly less trouble to make than the trypsinized pea extract. It has none of the disadrantages mentioned above. It. is beautifully clear and transparent,

* It is immaterial whether the patient is being treated or not with

arsenic or mercury.
$t$ I usually get 6 to 7 c.cm. of plasma from each centrifugal tube. 